BULL. AUSTRAL. MATH. SOC.

VOL. $5(1971), 187-189$.

\title{
A Banach space with support homeomorphism is reflexive
}

\author{
J.R. Giles
}

\begin{abstract}
For every Banach space $X$ there is a natural non-linear mapping from $X$ into its dual $X^{*}$. It is shown that if this mapping is a homeomorphism then it is onto $X^{*}$ and $X$ is reflexive.
\end{abstract}

In the study of the geometry of normed linear spaces the following mapping is of considerable significance.

DEFINITION. A mapping $x \rightarrow f_{x}$ of a normed linear space $X$ into its dual $X^{*}$ is called a support mapping if, for each $x \in\{x \in X:\|x\|=1\}$ and real $\lambda \geq 0, f_{x} \in\left\{f \in X^{*}: f(x)=\|f\|=1\right\}$ and $f_{\lambda x}=\lambda f_{x}$. (The Hahn-Banach Theorem guarantees that such a mapping exists for every normed linear space.)

NOTATION. We denote by $P$ the set of continuous linear functionals on a normed linear space $X$ which attain their norm on the unit sphere $\{x \in X:\|x\|=1\}$.

It is known that if the dual $X^{*}$ of a Banach space $X$ has a continuous support mapping then $X$ is reflexive, [3, Theorem 2]. The author has given the improved result that if the dual $X^{*}$ of a Banach space $X$ has a support mapping continuous on the linear span of $P$ then $X$ is reflexive, [4, Theorem $\left.1^{*}\right]$. Counter-examples have been display.ed to show that a Banach space with a continuous support mapping is not necessarily reflexive, and a Banach space $X$ whose dual $X^{*}$ has a support mapping continuous on $P$ is not necessarily reflexive, [4]. This note contributes to the study of the implications of continuity properties of

Received 15 March 1971. 
the support mapping.

Klee [5, p. 35] proposed the problem of characterizing those Banach spaces $X$ which are homeomorphic to their duals $X^{*}$ under a support mapping. The following theorem reduces Klee's problem to that of characterizing those Banach spaces which have a support homeomorphism, for it implies that the range of a support homeomorphism on a Banach space $X$ is always $X^{*}$.

THEOREM. A Banach space $X$ is reflexive if it has a support mapping which is a homeomorphism.

Proof. Let $x \rightarrow f_{x}$ be the support homeomorphism of $X$ into $X^{*}$. From the continuity of the support mapping on $X$ it follows that there is only one possible support mapping on $X$ and the range of the support mapping is $P,[3$, Lemmas 1 and 2]. Consider $X$ with metric

$$
d(x, y)=\frac{1}{2}\left\{\|x-y\|+\left\|f_{x}-f_{y}\right\|\right\},
$$

and $P$ with metric

$$
d^{*}\left(f_{x}, f_{y}\right)=\frac{1}{2}\left\{\|x-y\|+\left\|f_{x}-f_{y}\right\|\right\}
$$

$P$ with the $d^{*}$-topology is homeomorphic to $X$ with the $d$-topology under the support mapping. Since the support mapping is continuous on $X$, we have from [4, Lemma 2] that the d-topology is compatible with the linear structure on $X$. By the Metrisation Theorem for linear topological spaces, there exists an invariant metric on $X$ which generates the d-topology. Since support mappings are norm preserving, the balls centered on 0 for the $d$-metric are the same as those for the norm. Therefore the invariant metric which generates the $d$-topology is that induced by the norm. But we are given that $X$ with the norm-topology is homeomorphic to $P$ with the norm-topology under the support mapping. Therefore $P$ with the $d^{*}$-topology is homeomorphic to $P$ with the norm-topology under the identity mapping. That is, on $P$ the $d^{*}$-topology is the norm-topology. However, since $X$ is a Banach space, we have by [4, Lemma 3] that $P$ is complete in the $d^{*}$-topology. Therefore $P$ is complete in the norm-topology. But for every Banach space $X, P$ is norm-dense in $X^{*}$, [1], so it follows that $P=X^{*}$, and from [3, Theorem 2] that $X$ is reflexive. 
Cudia [2, p. 306] has given a solution to Klee's problem using the support mapping characterization of differentiability of the norm, [3, Theorem I (ii)]. Using our theorem we can derive the following improved solution which gives us further information about the relation between continuity of the support mapping and strong differentiability of the norm.

COROLLARY. The norms of a Bonach space $X$ and $i$ ts dual $X^{*}$ are strongly differentiable on their unit spheres if and only if there exists a support mapping on $X$ which is a homeomorphism.

It should be noted that our theorem is true for Banach spaces but not necessarily true for normed linear spaces. Every inner product space $X$ has an additive support mapping and since support mappings are norm preserving an additive support mapping is a homeomorphism. But not all inner product spaces are reflexive.

\section{References}

[1] Errett Bishop and R.R. Phelps, "A proof that every Banach space is subreflexive", Bulz. Amer. Math. Soc. 67 (1961), 97-98.

[2] Dennis F. Cudia, "The geometry of Banach spaces. Smoothness", Trans. Amer. Math. Soc. 110 (1964), 284-314.

[3] J.R. Giles, "On a characterisation of differentiability of the norm of a normed linear space", J. Austral. Math. Soc. 12 (1971), $106-114$.

[4] J.R. Giles, "On a differentiability condition for reflexivity of a Banach space", J. Austral. Math. Soc. (to appear).

[5] Victor L. Klee, Jr, "Convex bodies and periodic homeomorphisms in Hilbert space", Trans. Amer. Math. Soc. 74 (1953), 10-43.

The University of Newcastle, Newcastle, New South Wales. 